\title{
Tunable fragmentation of organic molecules in laser ablation glow discharge time-of-flight mass spectrometry
}

\section{Journal Article}

Author(s):

Lotito, G.; Günther, Detlef

Publication date:

2012

Permanent link:

https://doi.org/10.3929/ethz-b-000047061

Rights / license:

In Copyright - Non-Commercial Use Permitted

Originally published in:

Analytical and Bioanalytical Chemistry 402(8), https://doi.org/10.1007/s00216-011-5498-x 


\title{
Tunable fragmentation of organic molecules in laser ablation glow discharge time-of-flight mass spectrometry
}

\author{
G. Lotito • D. Günther
}

Received: 24 August 2011 /Revised: 7 October 2011 / Accepted: 11 October 2011 /Published online: 27 October 2011

(C) Springer-Verlag 2011

\begin{abstract}
A DC-pulsed glow discharge (GD) has distinct temporal regimes which are characterized by "softer" or "harder" ionization of analytes introduced into the discharge. It is thus possible to obtain both molecular weight and structural fragment information from the same spectra. In order to extend the capabilities of this technique a laser ablation (LA) sampling system was coupled to a DC-pulsed GD and to a time-of-flight (TOF) mass spectrometer (MS) for characterizing organic samples such as oleic acid, reserpine, two different peptides, and a polymer. Both hard and soft ionization regimes were studied. These LAGDTOFMS results were compared to matrix-assisted laser desorption ionization (MALDI) spectra using the same compounds (i.e., analytes, concentration, and matrix). It was found that LAGD offers tunable ionization and provides a reduced matrix dependence. However, the sensitivity achieved by the prototype LAGD-TOFMS was significantly lower when compared with commercially available MALDI-TOFMS instrumentation. Since LAGDTOFMS is rather new, some technical details to increase its sensitivity are discussed.
\end{abstract}

Keywords Pulsed glow discharge $\cdot$ MALDI $\cdot$ Laser ablation $\cdot$ Fragmentation $\cdot$ Chemical speciation

Published in the special issue Analytical Sciences in Switzerland with guest editors P. Dittrich, D. Günther, G. Hopfgartner, and R. Zenobi.

G. Lotito $\cdot$ D. Günther $(\bowtie)$

Laboratory of Inorganic Chemistry,

ETH Zurich,

8093, Zurich, Switzerland

e-mail: guenther@inorg.chem.ethz.ch

\section{Introduction}

A wide variety of applications demonstrate a growing need for complete sample characterization, which includes in addition to the elemental concentrations, the analysis of the chemical species and its concentration $[1,2]$. Many applications of chemical speciation can be found in environmental sciences, for instance, for the interpretation of chemodynamic properties and toxicity of chemical compounds [3], and in biomedical research [4]. Chemical speciation is commonly achieved by using hyphenated techniques, where a chromatographic separation procedure and an ionization source to identify the given element/molecule in a mass spectrometer are combined. These ionization sources can either be hard-ionization sources providing elemental information such as the inductively coupled plasma $[5,6]$ or soft ionization sources such as electrospray ionization, chemical ionization, or matrix-assisted laser desorption ionization (MALDI) [7, 8] which provide molecular weight information. There are approaches to combine these techniques within one instrument $[9,10]$. However, the instruments do not generate all the information from the same sample volume. Therefore, a single ionization source providing all the required information simultaneously or quasisimultaneously would be beneficial.

A pulsed glow discharge (GD), which is used in the trace elemental analysis of bulk solid samples [11] has the potential to overcome some of the shortcomings of other techniques. By using a pulsed GD, it is possible to achieve a high instantaneous pulse power, avoiding over heating of the cathode [12-14] and generating different temporal regimes $[15,16]$. These regimes, prepeak, plateau, and afterglow, provide different information about the composition of a sample [17]. Ionization during the prepeak is 
dominated by electron ionization (EI), whereas the plateau regime consists of a mixture of EI, Penning ionization (PI) and charge exchange processes. However the main process is assumed to be EI, which occurs when an atom or molecule collides with an electron whose energy is higher than the ionization energy of the atom, according to the reaction:

$M^{0}+e^{-} \rightarrow M^{+}+2 e^{-}$

where $M^{0}$ is the atom or molecule in the ground state, and $M^{+}$is the atom or molecule in an ionized state. After termination of the pulsed GD, in the afterglow regime, the main ionization process is PI which occurs as the result of collisions between metastable gas atoms and atomic or polyatomic species which have an ionization energy lower than the energy of the excited metastable atom. Argon metastable states with energies of 11.55 and $11.72 \mathrm{eV}$ have been observed in a GD and are sufficient to ionize elements and molecules. A typical PI reaction is for example:

$M^{0}+\mathrm{Ar}^{*} \rightarrow M^{+}+\mathrm{Ar}^{0}+e^{-}$

where $\mathrm{Ar}^{0}$ is the ground state argon atom and $\mathrm{Ar}^{*}$ is the argon atom in a metastable state. For instance, Steiner et al. [17] used a pulsed DC GD TOFMS for sequential elemental and molecular analysis of volatile samples. In these studies, a small amount of $p$-xylene was introduced into a GD plasma. During the prepeak and the plateau regime - in addition to the discharge gas ions - several fragments were detected. The mass spectra acquired in the afterglow showed intact parent molecules of the analyte and no major fragmentation was reported, which is an indication of a soft ionization process. In another report by Lewis et al. [18], the successful analysis of aromatic compounds such as $o$-xylene using a pulsed GD-TOFMS coupled to gas chromatography (Ar as the carrier) was reported. A pulsed GD coupled to a gas chromatography system was also reported by Fliegel et al. [19], where elemental and molecular information from gaseous analytes such as toluene in methanol as solvent was demonstrated. In addition, Majidi et al. [16] showed the possibility of performing the chemical speciation of volatile compounds using a $\mu$ s-pulsed GD-TOF system. The analysis of solid samples has been reported by Tarik et al. [20] where organic compounds including benzoic acid, vanillic acid, Teflon, and caffeine have been introduced into the GD-TOFMS by laser ablation. In that study elemental ions were observed in the plateau. The afterglow contained information about the parent molecule. Further examples can be found in the literature [21, 22] indicating the growing interest in pulsed GD for chemical speciation. In addition, radio frequency GD sources operating in a continuous mode have also shown to provide simultaneous molecular and structural information when coupled to gas chromatography [23] as well as to liquid chromatography [24].

In this study, a laser ablation system coupled to a pulsed GD-TOFMS (LAGD-TOFMS) was used to investigate the soft and hard ionization capabilities of this technique by time-resolved injection of various organic compounds, such as oleic acid, reserpine, two peptides, and a polymer. As well, the samples for LAGD-TOFMS were using matrixes commonly used in MALDI. These experiments were specifically designed to separate the desorption and ionization steps in time and space. Another aim of this work was to determine the performance of this technique complementary to MALDI [25, 26], which represents a state-of-the-art technique for the analysis of organic materials. Therefore, the selection of compounds was focused on low molecular weight samples, which is not the major application of this otherwise widely applied technique. In order to provide comparable results, the same compounds were analyzed with parameters as closely matched as possible in the two techniques.

\section{Experimental}

Figure 1 shows the schematic experimental setup of both techniques used in this study. The LAGD-TOFMS system (Fig. 1a) has been described in detail elsewhere [27]. An $\mathrm{N}_{2}$ $(337 \mathrm{~nm})$ laser (Spectra-Physics, CA, USA) with a pulse width of approximately $5 \mathrm{~ns}$ was used to desorb the sample. The laser energy was optimized by using an energy density filter wheel to generate pulse energies of either 19 or $190 \mu \mathrm{J}$. This set of energies was sufficient to generate signals from all samples studied. The mass analyzer was a commercial orthogonal extraction TOF system (H-TOF from Tofwerk AG, Thun). The spectra were acquired in positive ion mode and each result consists of a mean of 10 laser shots onto the same sample position. Table 1 gives a summary of the operating conditions used in this study. Figure $1 \mathrm{~b}$ shows the experimental set-up of the MALDITOFMS system (Bruker Daltonics Ultraflex II, MA, USA), which was used for comparison. The sample plate was placed at a distance of $2 \mathrm{~mm}$ in front of the first extraction grid which was then placed $3 \mathrm{~mm}$ from the second extraction grid. This configuration was used in order to maximize the sampling efficiency of the LA plume. The instrument was furthermore equipped with an observation optic to visualize the sample surface. The measurements were carried out using the same wavelength as used for LAGD-TOFMS $(337 \mathrm{~nm})$. The laser energy was adjusted between 19 and $30 \mu \mathrm{J}$ per pulse, which represents typical 
Fig. 1 Experimental setup of: (a) the LAGD-TOFMS instrument, where $a$ is the cathode-anode distance equal to $10 \mathrm{~mm}, b$ is the distance between the sample surface and the axis connecting the cathode to the sapling orifice equal to $4 \mathrm{~mm}$, and $c$ is the diameter of the surface over which the sample is spread and is equal to $5 \mathrm{~mm}$; (b) the MALDI-TOFMS Bruker Daltonics Ultraflex II instrument

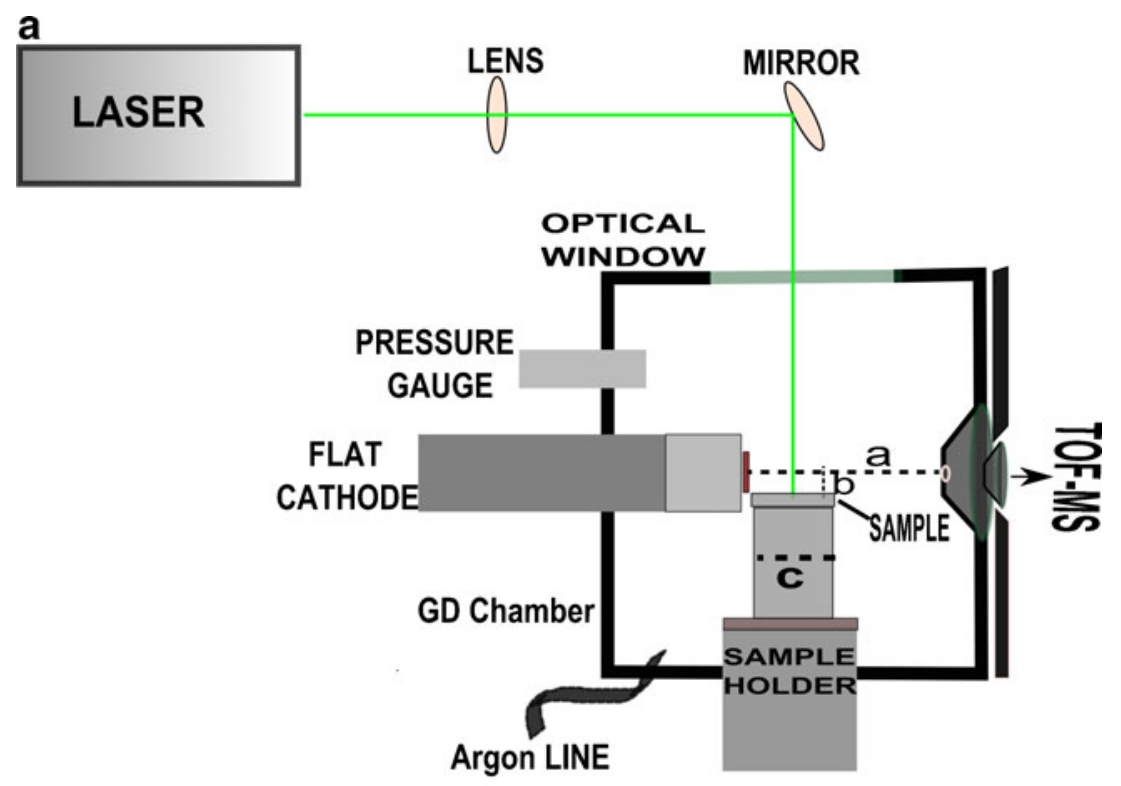

b

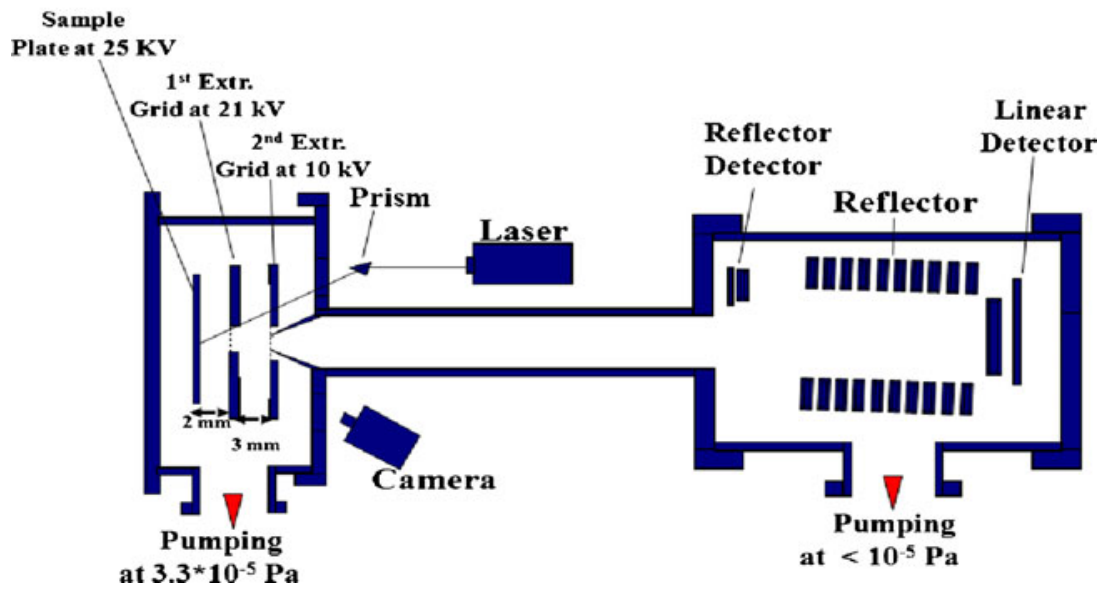

values for desorption of these types of samples. The spectra, measured in positive ion mode, were obtained by integrating approximately 500 laser shots placed across the sample. Table 2 contains the operating conditions used in this study.

\section{Sample preparation}

The samples were prepared using the protocol commonly used for MALDI. The analytes were mixed in ratios ranging from $1 \%$ to $50 \% \mathrm{~mol} / \mathrm{mol}$ with the absorbing matrix, in this work alpha-cyano-4-hydroxycinnamic acid (CHCA, $\mathrm{C}_{10} \mathrm{H}_{7} \mathrm{O}_{3} \mathrm{~N}, M=189.04 \mathrm{~g} / \mathrm{mol}$ ) was used (see supplementary information in Appendix A.1, Fig. 12). Solutions of CHCA were prepared by dissolving $20 \mathrm{mg}$ in $700 \mu \mathrm{L}$ of acetonitrile and $100 \mu \mathrm{L}$ of $0.1 \%$ trifluoroacetic acid. Oleic acid $\left(\mathrm{C}_{18} \mathrm{H}_{34} \mathrm{O}_{2}, 282.26 \mathrm{~g} / \mathrm{mol}\right)$, reserpine $\left(\mathrm{C}_{33} \mathrm{H}_{40} \mathrm{~N}_{2} \mathrm{O}_{9}, 608.27 \mathrm{~g} / \mathrm{mol}\right)$, the peptide bradykinin fragment 1-7 $\left(\mathrm{C}_{35} \mathrm{H}_{52} \mathrm{~N}_{10} \mathrm{O}_{9}, 756.39 \mathrm{~g} / \mathrm{mol}\right)$, the peptide GlyArg-Gly-Asp $\left(\mathrm{C}_{14} \mathrm{H}_{25} \mathrm{~N}_{7} \mathrm{O}_{7}, 403.18 \mathrm{~g} / \mathrm{mol}\right)$, and a polyethylene glycol 600 polymer with a repeating unit of $-\mathrm{C}_{2} \mathrm{H}_{4} \mathrm{O}$ (44 $\mathrm{g} / \mathrm{mol})$ were selected as analytes. All chemicals were purchased from Sigma Aldrich (St. Louis, MO).

\section{Results and discussion}

To determine the capabilities of LAGD-TOFMS in comparison to state-of-the-art MALDI, a selection of analytes (oleic acid, reserpine, peptide bradykinin fragment 1-7, peptide Gly-Arg-Gly-Asp, peg 600) were measured by both techniques. The concentration of the analytes was adapted to the current sensitivity of our prototype LAGD-TOFMS 
Table 1 Experimental parameters regarding the LAGD-TOFMS setup

\begin{tabular}{ll}
\hline Parameters & Values \\
\hline GD & \\
Carrier gas, purity & Ar, 6.0 \\
Type of cathode and element & Flat $(\mathrm{Ta})$ \\
Pressure in the source $(\mathrm{Pa})$ & $36-45$ \\
Voltage anode-cathode $(\mathrm{kV})$ & -1 \\
Distance anode-cathode $(\mathrm{mm})$ & 10 \\
GD pulse on-time $(\mathrm{ms})$ & 1.5 \\
Pulse period $(\mathrm{ms}) /$ frequency $(\mathrm{Hz})$ & $50 / 20$ \\
Sampler aperture $(\mathrm{mm})$ & 1 \\
Sample-axis sampler distance $(\mathrm{mm})$ & 4 \\
Laser & \\
Type & $\mathrm{N}_{2}$ \\
Wavelength $(\mathrm{nm})$ & 337 \\
Frequency $(\mathrm{Hz})$ & 20 \\
Pulse energy $(\mu \mathrm{J})$ & $19-190$ \\
Spot size $(\mu \mathrm{m})$ & $30-70$ \\
Focal length $(\mathrm{cm})$ & 35 \\
Irradiance $\left(\mathrm{W} / \mathrm{cm}^{2}\right)$ & $6.72 \times 10^{9}-1.23 \times 10^{9}$ \\
Fluence $\left(\mathrm{J} / \mathrm{cm}^{2}\right)$ & $2.69-4.94$ \\
\hline
\end{tabular}

even knowing that the sensitivities of current state-of-theart MALDI instruments provide access to much lower concentrations. However, the results discussed below are rather focused on the different information achievable by each technique. To make the comparison as meaningful as possible, same analyte concentrations were analyzed by both techniques.

Oleic acid A mixture of oleic acid in CHCA $(50 \% \mathrm{~mol} /$ mol) was desorbed and subsequently detected in the different time regimes of the pulsed GD. Three representative mass spectra of each of the temporal regimes

Table 2 Experimental parameters used in the MALDI-TOFMS setup

\begin{tabular}{ll}
\hline Parameters & Values \\
\hline Sample plate voltage $(\mathrm{kV})$ & 25 \\
1st Extr. Grid $(\mathrm{kV})$ & 21 \\
2nd Extr. Grid $(\mathrm{kV})$ & 10 \\
Pressure in the source $(\mathrm{Pa})$ & $10^{-5}$ \\
Laser & \\
Type & $\mathrm{N}_{2}$ \\
Wavelength $(\mathrm{nm})$ & 337 \\
Frequency $(\mathrm{Hz})$ & 50 \\
Pulse energy $(\mu \mathrm{J})$ & $19-30$ \\
\hline
\end{tabular}

containing mass spectra of GD only, matrix only (both used as blank for checking interferences), and matrix plus analyte are shown in Fig. 2. The prepeak regime shows significant molecular ion signal intensities from the matrix (shown in the spectra of matrix only and matrix plus analyte), but no other significant signals. The mass spectra of the plateau regime are similar to the prepeak. However, the matrix plus analyte spectra reveal several peaks (two fragments, the protonated and the molecular peak) which can be attributed to the analyte, even though their intensities are low.

In contrast, the afterglow spectra contained the molecular ion and protonated peak which is approximately 30 times higher than those measured in the plateau. This is also observed for the fragments $(\mathrm{m} / \mathrm{z} 264$ and 265 identifiable resp. with the bond cleavage of $\mathrm{H}_{2} \mathrm{O}$ and hydroxyl group present in the oleic acid molecule) and could be explained by the fact that during the plateau of the pulsed GD a greater fragmentation due to the EI of the molecule takes place. In the case of the prepeak (upper part in Fig. 2), the signal coming from the analyte is close to the background intensity, which might be due to an even stronger fragmentation of the oleic acid's molecule during this temporal regime.

The same compound (oleic acid $50 \% \mathrm{~mol} / \mathrm{mol}$ in CHCA) was measured using MALDI and the same mass range, as shown for LAGD-TOFMS (see Fig. 2), is summarized in Fig. 3.

The entire MALDI spectra (see Fig. 13 in Appendix A.2) are dominated by peaks originated from the matrix. However, the MALDI spectra $(m / z=188-287)$ shown in Fig. 3 do not contain protonated and molecular peaks for oleic acid in CHCA. The reason for the absence of the analyte peaks might be caused by inefficient ionization of oleic acid molecules by CHCA, which has been observed in MALDI of fatty acids (FA) [28, 29]. There it has been described that the direct detection of FA using a common matrix such as 2.5 DHB (2,5-dihydroxybenzoic acid) or CHCA was not observed. As alternative, the use of dimethylamino-naphthalene has been successfully used for detecting FA [30]. This type of matrix belongs to a class of compounds described as "proton sponges" due to their ability to take up available protons. This is due to their very high basicity ( $\mathrm{p} K_{\mathrm{a}} 12.1$ in water), which allows, in the example of fatty acids, the matrix to take up even weakly acidic protons from the carboxyl group, thus forming deprotonated anions which can then be detected in the negative ion mode. In contrast, the $\mathrm{p} K_{\mathrm{a}}$ of CHCA and DHB are 1.17 and 3.01 , respectively [31, 32]. In the case of the LAGD, the ionization is not assisted by the matrix and is mainly stimulated by the Ar metastable excited atoms in the afterglow or the free electrons in the powering phase of the pulsed GD. This indicates a less 
Fig. 2 Comparison between spectra in the prepeak (top), plateau (middle), and afterglow (bottom) acquired from the pulsed GD (GD only) blank, the pulsed GD and a sample containing only the matrix (CHCA only), and the pulsed GD and the sample containing the matrix and the analyte (oleic acid)

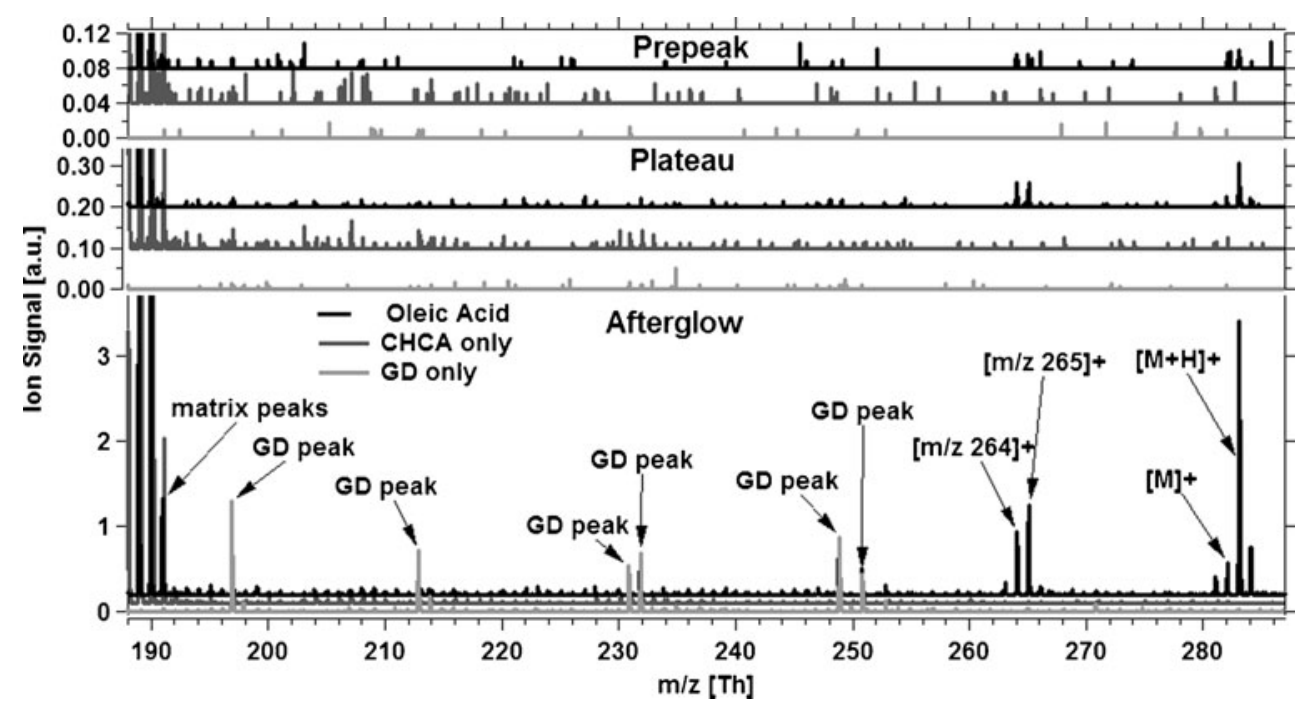

matrix-dependent behavior of the ionization process in LAGD-TOFMS compared to MALDI.

Figure 4 shows the spectra of oleic acid in the low mass region during the powering phase (bottom part) for the background (GD only), the matrix (CHCA only), and the matrix containing the analyte (oleic acid). Comparing the fragmentation pattern of LAGD-TOFMS with the EI mass spectrum as published in the NIST web database [33], it is observed that this technique provides important information about the structure. For example, the methylene group's fragmentation is distinguishable in the powering phase from the matrix and the background of the GD. However, some of the peaks occurring for the matrix only are not detected when analyzing the matrix with analyte, which might be related to the sensitivity. But it is important to note that the powering phase provides far less interferences, which allows access to the structural information. Some of the peaks detected in GD only might be caused by the presence of $\mathrm{H}_{2} \mathrm{O}^{+}$and $\mathrm{H}_{3} \mathrm{O}^{+}[34,35]$ ions. For example, the $\mathrm{m} / \mathrm{z} 55$ (one of the ions corresponding to a fragment from oleic acid) can be identified as $\left(\left(\mathrm{H}_{2} \mathrm{O}\right)_{3} \mathrm{H}\right)^{+}$. Nevertheless, during the powering phase there are peaks at $m / z=83,97$, and $111 \mathrm{~m} / z$ which are not significantly affected by interferences. The spectra in the afterglow (Fig. 4, upper part) show a much higher contribution of the interferences in this temporal regime when compared to the powering phase. Therefore it is recommended that the structural information be retrieved from the powering phase. The observation of more interferences in the afterglow might be caused by water clusters due to PI, while during the powering phase these molecules are highly fragmented by EI.

The spectrum shown in Fig. 4 was acquired using a concentration of $50 \% \mathrm{~mol} / \mathrm{mol}$ oleic acid and is representative for all other mixtures studied (with concentrations ranging down to $10 \%, \mathrm{~mol} / \mathrm{mol}$ ). From all experiments, it
Fig. 3 Spectra of oleic acid $50 \%$ in CHCA measured with the MALDI-TOFMS

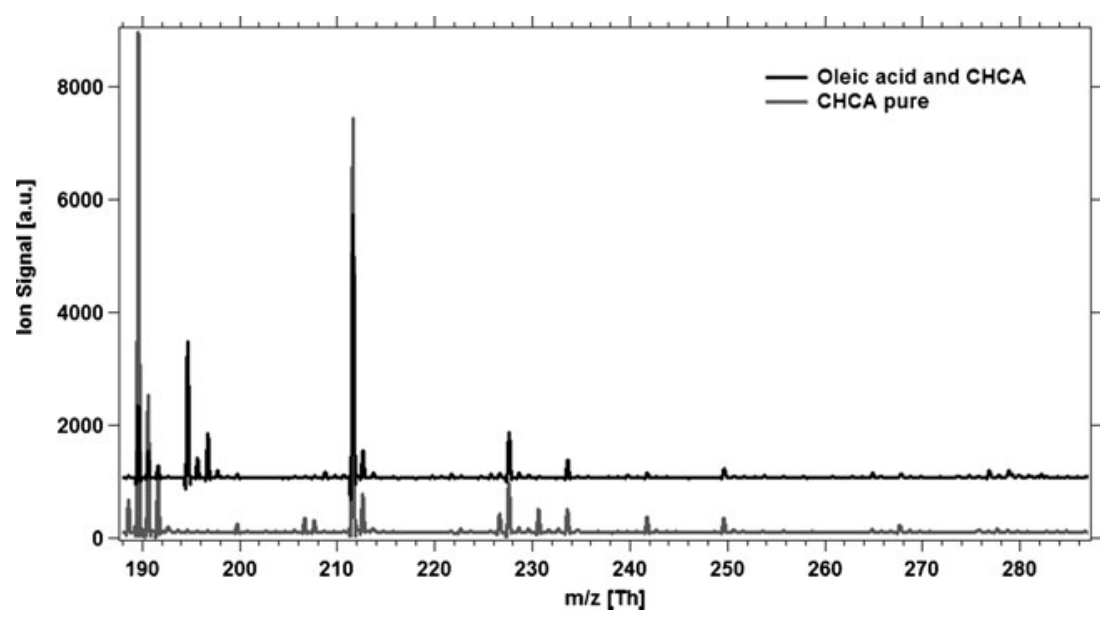


Fig. 4 Comparison of the spectra of oleic acid (along with those from the matrix and the GD) during the afterglow and the powering phase of the pulsed GD in the low mass region

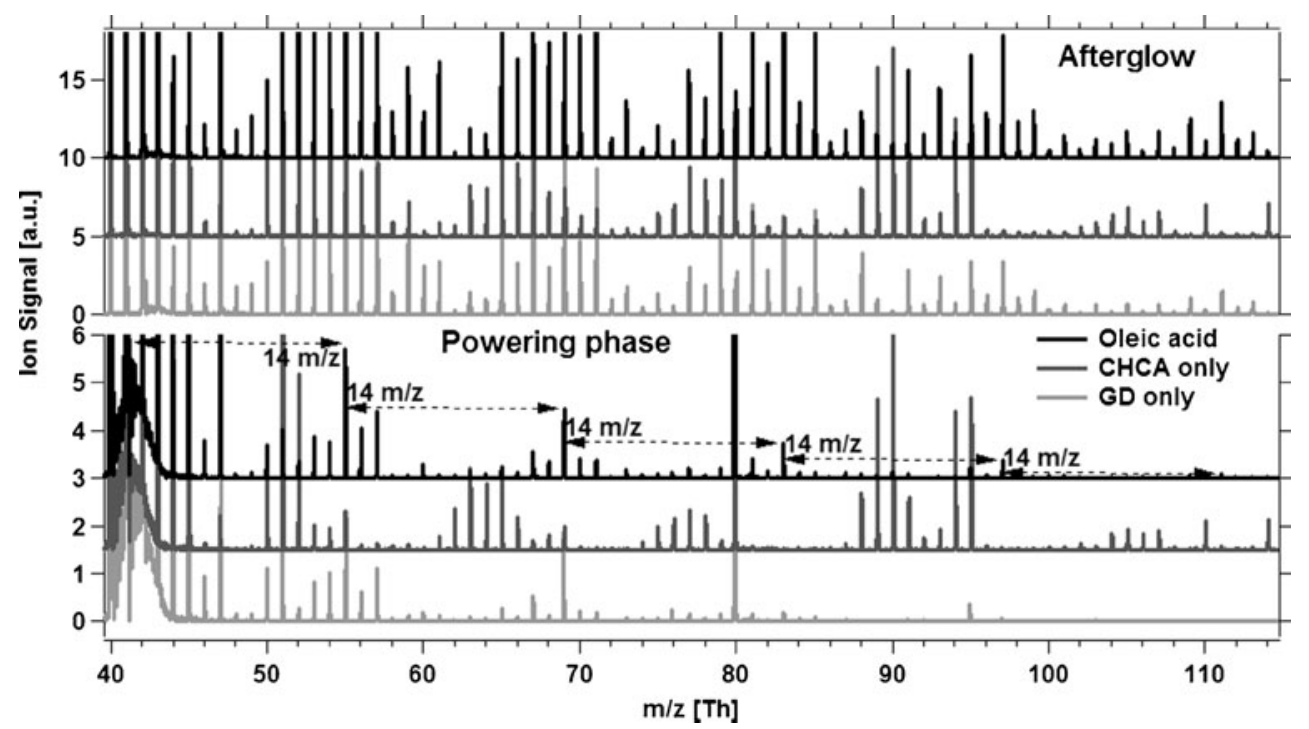

can be concluded that the afterglow signal from the molecular ion and its protonated form is always higher than in the powering phase, which demonstrates that a stronger fragmentation in the powering phase takes place. Furthermore it has been observed that the intensity of the signal of the molecular ion and its protonated form in MALDI-TOFMS was always at background level.

Reserpine A series of mixtures containing 1\%, 10\%, and $50 \% \mathrm{~mol} / \mathrm{mol}$ reserpine in CHCA were analyzed in the different time regimes (as done for oleic acid) of the pulsed GD. For this compound, an analyte to matrix ratio of $50 \%$ was required to generate signals clearly above background. For a direct comparison of the two techniques, we measured the same sample concentrations by MALDI, even knowing that these concentrations are unusually high for MALDI.

In Fig. 5 are shown the spectra generated in the three temporal regimes of the GD. Figure 5 shows the spectrum of the afterglow regime of reserpine with the molecular ion peak and some other analyte peaks (see assignment in Table 3). When compared with the EI mass spectrum of this compound [33], these peaks can be identified as fragments from the analyte. The presence of these fragments in the afterglow, which is mainly characterized by PI, can be generated by the laser ablation processes itself, as discussed in the literature [20]. There, the appearance of organic fragments (benzoic acid, polytetrafluoroethylene, polyvinyl chloride) during ablation of these materials into the afterglow has been shown. In conclusion, the molecular
Fig. 5 Comparison between different spectra in the prepeak (top), plateau (middle), and afterglow (bottom) acquired from the pulsed GD background (GD only), the pulsed GD with a sample containing only the matrix (CHCA only), and the pulsed GD with a sample lcontaining the matrix and the analyte (reserpine)

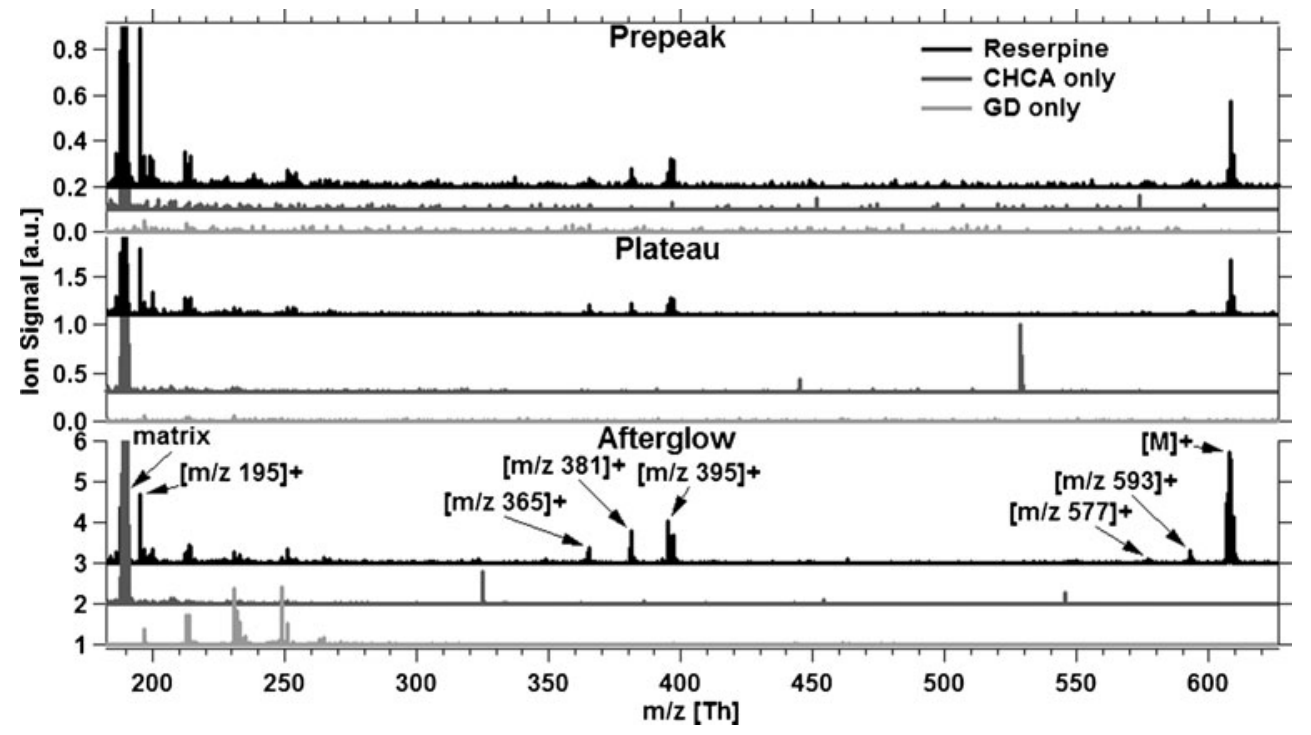


Table 3 Possible assignments of fragments of reserpine

\begin{tabular}{ll}
\hline Peak $(m / z)$ & Assignment \\
\hline 593 & $\mathrm{M}-\mathrm{CH}_{3}$ \\
577 & $\mathrm{M}-\mathrm{CH}_{3}-\mathrm{CH}_{3}-\mathrm{H}$ \\
395 & $\mathrm{C}_{23} \mathrm{H}_{27} \mathrm{~N}_{2} \mathrm{O}_{4}$ \\
381 & $m / z 395-\mathrm{CH}_{3}+\mathrm{H}$ \\
365 & $\mathrm{C}_{22} \mathrm{H}_{25} \mathrm{~N}_{2} \mathrm{O}_{3}$ \\
195 & $\mathrm{C}_{10} \mathrm{H}_{11} \mathrm{O}_{4}$ \\
\hline
\end{tabular}

ion was most sensitively detected in the afterglow regime. However, the molecular information was also generated in the other two temporal regimes (plateau and prepeak). For comparison, a spectrum acquired of the same sample using MALDI is shown in Fig. 6.

This spectrum (displayed in Fig. 6) shows the detection of the molecular ion without any interference from the matrix, which is called the matrix suppression effect (MSE). Experimental parameters relevant for this effect, such as laser intensity and the molar ratio of matrix and analyte in the sample have been reported [36]. Comparing the spectra shown in Figs. 5 and 6, it can be seen that the MALDI spectrum contains mainly the molecular ion whereas LAGD-TOFMS provides additional information about the analyte. In addition it provides an indication that ionization in LAGD is less matrix-dependent when compared to MALDI, which could be beneficial for quantitative analysis. However, measurements with lower reserpine concentration (i.e., $1 \%$ and $10 \%$ ) indicate a significantly increased sensitivity and lower limits of detection for MALDI. This difference between the two techniques may be mainly due to the sampling geometry. Indeed, in MALDI the sample is placed directly in front of the sampling orifice at a distance of $c a .2 \mathrm{~mm}$ from the first extraction grid (c.f. Fig. 1b) and the generated ions are extracted and accelerated into the TOFMS. In LAGD the sample is perpendicular to the sampling orifice with a mean distance of $8.5 \mathrm{~mm}$ (c.f. Fig. 1a). As well, losses due to shorter mean free paths in the GD source should be taken into account. Thus, a higher ion loss occurs within the GD chamber. Furthermore, the sample holder is grounded. These current limitations need to be reduced to give more powerful quantification capabilities.

Peptides A series of mixtures containing two different peptides, the bradykinin fragment 1-7 and the Gly-ArgGly-Asp in CHCA, in $1 \%, 10 \%$, and $50 \% \mathrm{~mol} / \mathrm{mol}$ ratios were also analyzed in the different time regimes of the pulsed GD. Only at an analyte concentration of $50 \%$ within CHCA and by using a laser energy of $190 \mu \mathrm{J}$ was it possible to detect both of the peptides (Figs. 7 and 8). Comparing the spectra generated within the different time regimes indicates that the same ionization processes (PI and EI) as discussed for oleic acid and reserpine take place. Furthermore it needs to be mentioned that the plateau (bradykinin, see Fig. 7) provides more information than the afterglow. It is also noted that the reproducibility of those measurements was strongly dependent on the laser sampling position. In some experiments there was no detection of the analyte, mainly due to inhomogeneity of the crystal structure of the matrix and the analyte; such inhomogeneity gives rise to regions of different density [37-39], which is a common problem reported in MALDI sample preparation.

Measurements of bradykinin by MALDI show mainly the detection of the protonated molecular ion due to MSE (only one protonated peak, data not shown). For the other peptide (Fig. 9), the protonated molecular ion and one adduct were observed, whereas the other peaks are from the matrix. For both peptides, there is no presence of other fragments. However, the sensitivity of the protonated molecular ion was much higher using MALDI than by LAGD due to the reasons mentioned above.
Fig. 6 Spectra of reserpine $50 \%$ with CHCA taken with MALDI-TOFMS

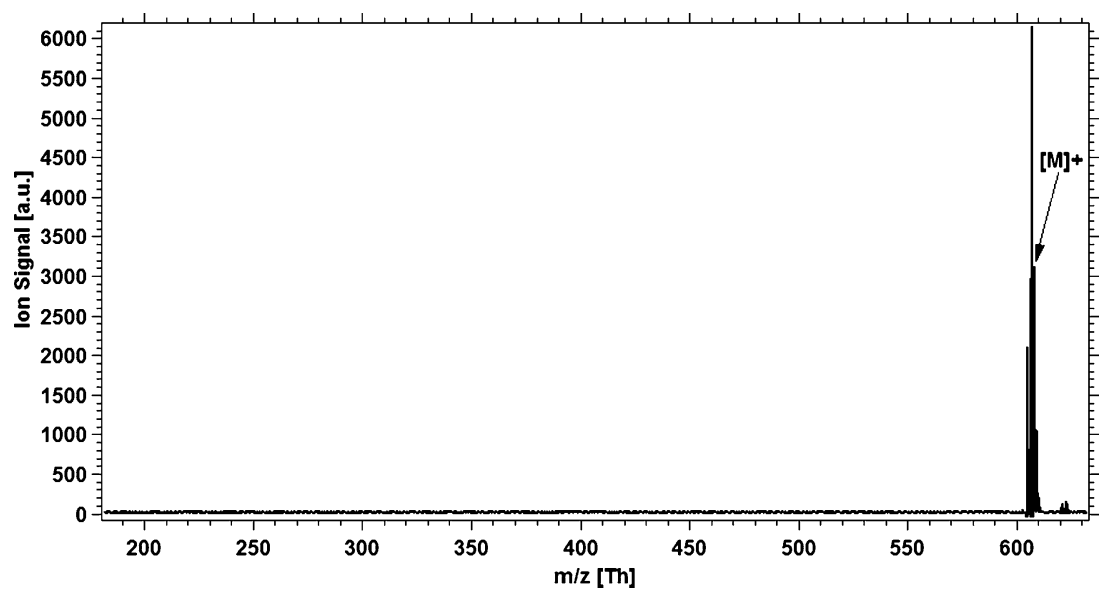


Fig. 7 Comparison of different spectra acquired from bradykinin $50 \%$ with CHCA in the prepeak (top), plateau (middle), and afterglow (bottom) acquired from the pulsed GD background (GD only), the pulsed GD with a sample containing only the matrix (CHCA only), and the pulsed GD with a sample containing the matrix and the analyte (bradykinin)

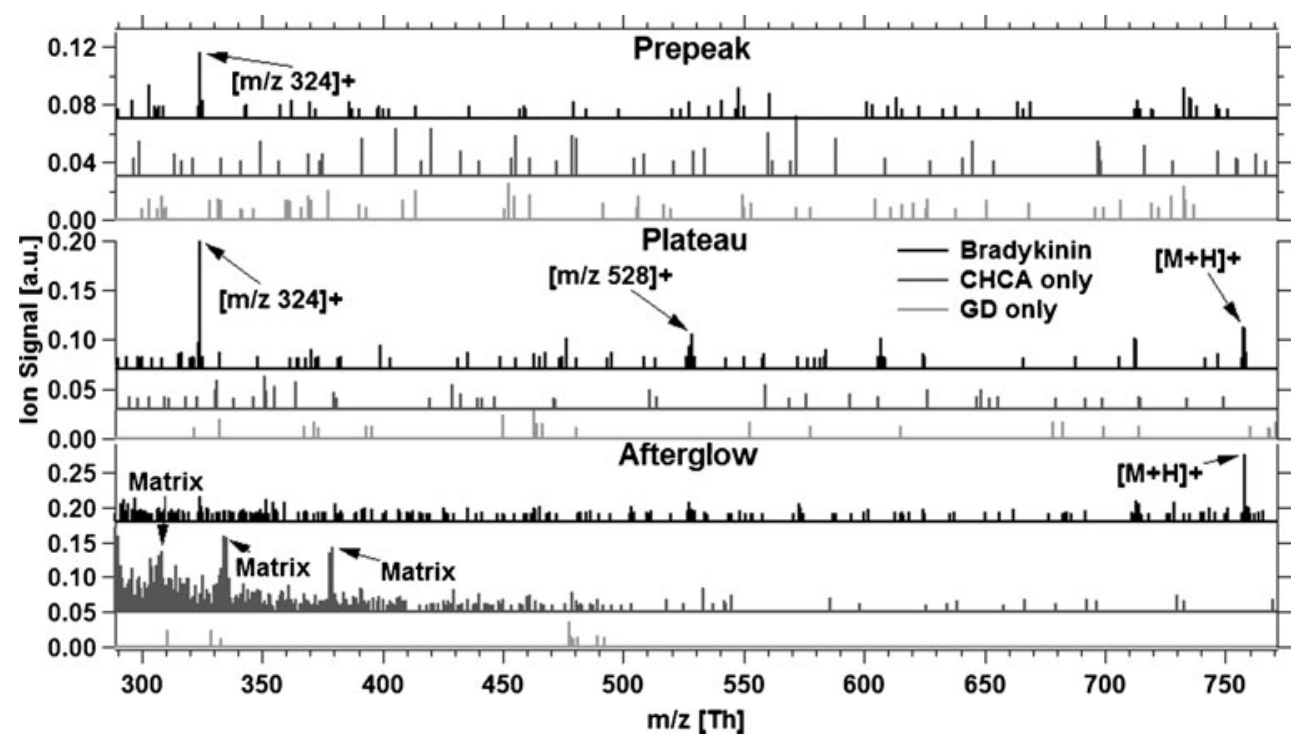

Polymer PEG 600 Another class of compounds was analyzed, the polymer polyethylene glycol 600 (PEG 600) in $\mathrm{CHCA}$ at $1 \%, 10 \%$, and $50 \% \mathrm{~mol} / \mathrm{mol}$ ratios, in the different time regimes of the pulsed GD. Significant detection above background of the analyte was achieved at concentrations of $10 \%$ and higher when using laser energies between 60 and 190 uJ. Figure 10 contains a summary of spectra (high and low $m / z$ ) acquired using PEG $60050 \% \mathrm{~mol} / \mathrm{mol}$ mixed with CHCA. Each of the peaks appearing in the afterglow and labeled by an arrow in Fig. 10a is an adduct resulting from the bonding between a multiple of the repeating unit of the polymer $\left(-\mathrm{C}_{2} \mathrm{H}_{4} 0-, 44\right.$ $m / z$ ) and $\mathrm{a} \mathrm{Na}^{+}$cation, which was an impurity within the sample. Each of those adducts was separated by $44 \mathrm{~m} / \mathrm{z}$ from the previous or following adduct. In the lower mass range of the sprectra, the detection of other repeating units was possible even though some masses such as 45,89 , and $133 \mathrm{~m} / \mathrm{z}$ present interferences with the GD and the matrix.
Furthermore, the intensities of some of the repeating units in the lower mass range shown in Fig. 10b $(\mathrm{m} / \mathrm{z} 177$ and 221, which are not affected by interferences) are higher than the intensities of the adducts in the higher mass range. This could be explained by the fact that the lower mass range repeating units are better sampled because of higher velocity and diffusion which allows them to reach the sampling orifice more easily. However, processes involving collisions of the analyte particles with the plasma gas atoms could also induce vibrational excitation and perhaps dissociation and might also play a role. In the plateau the detection of the different adducts is visible even though their intensity was lower than during the afterglow as also observed for the other analytes. In the lower mass range, the detection of some of the repeating units is more easily interpreted than during the afterglow due to less interferences from the GD and matrix. In the prepeak, the intensity of the adducts in the higher mass range was at the
Fig. 8 Comparison between different spectra in the prepeak (top), plateau (middle), and afterglow (bottom) acquired from the pulsed GD background (GD only), the pulsed GD with a sample containing only the matrix (CHCA only), and the pulsed GD with a sample containing the matrix and the analyte (peptide Gly-Arg-Gly-Asp)

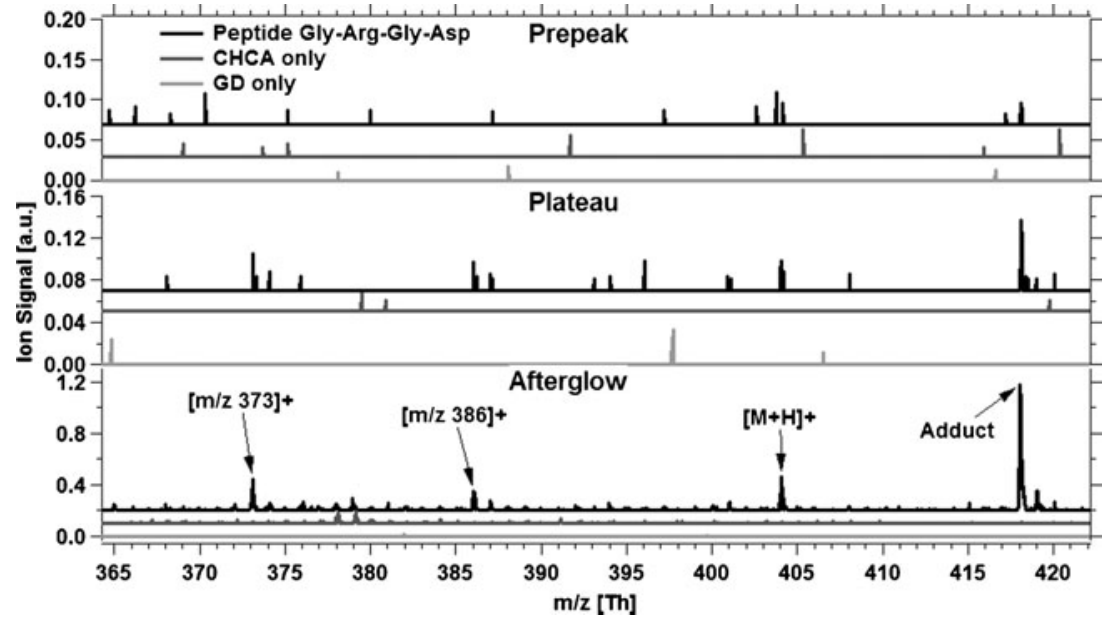


Fig. 9 Spectra of peptide GlyArg-Gly-Asp 50\% with CHCA (Peptide in CHCA) and CHCA only (CHCA pure) through MALDI

Fig. 10 Comparison between different spectra in the high mass range (a) and low mass range (b) in the prepeak (top), plateau (middle), and afterglow (bottom) acquired from the pulsed GD background (GD only), the pulsed GD with a sample containing only the matrix (CHCA only), and the pulsed GD with a sample containing the matrix and the analyte (PEG 600)
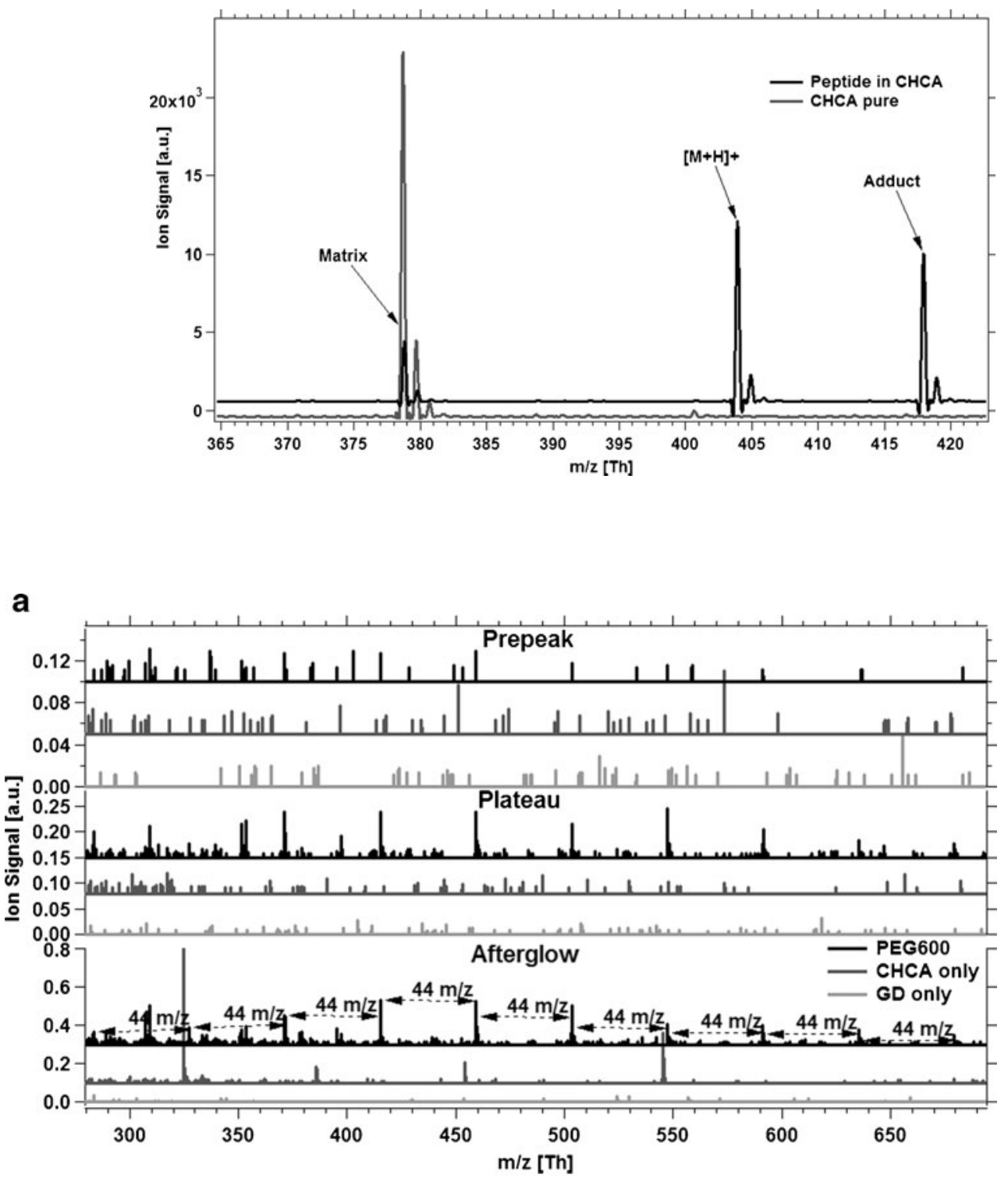

b

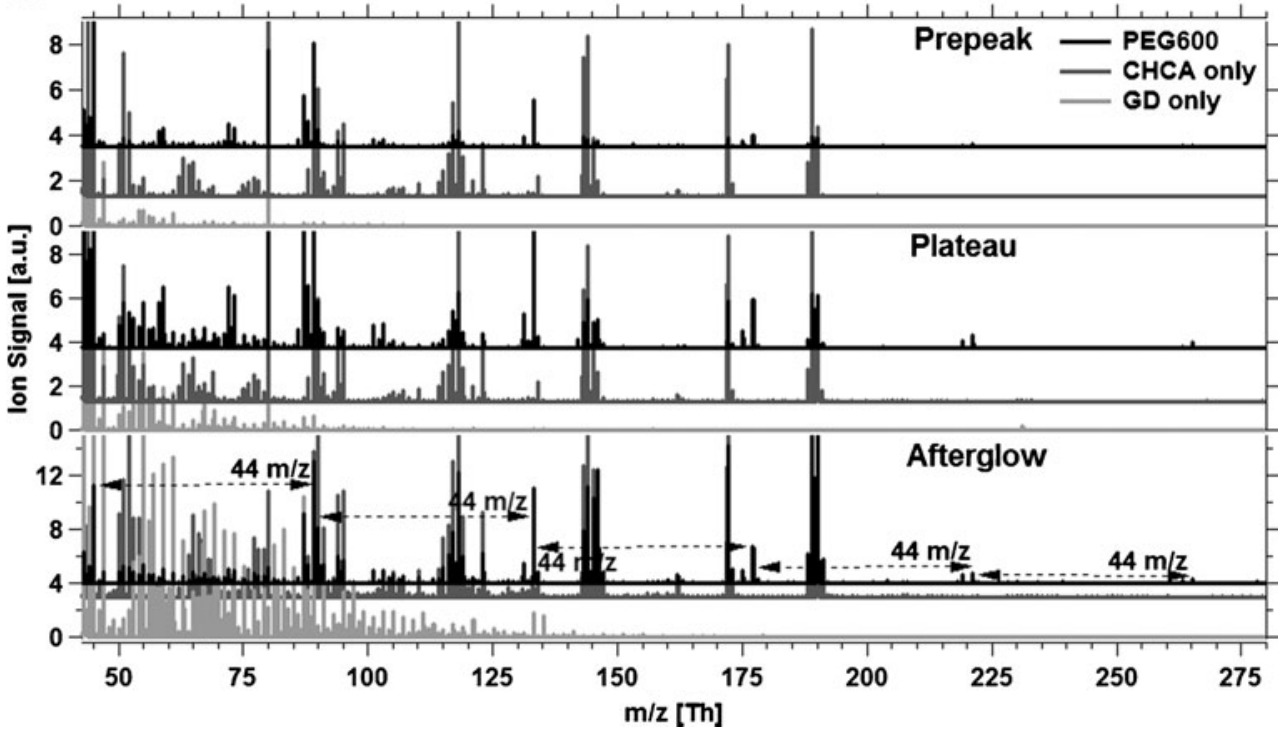


Fig. 11 Spectra of PEG 600 $50 \%$ with CHCA (PEG 600 in CHCA) and CHCA only (CHCA pure) with MALDI. The arrows indicate the constant difference between two repeating units of the polymer, i.e., 44 $\mathrm{m} / \mathrm{z}$

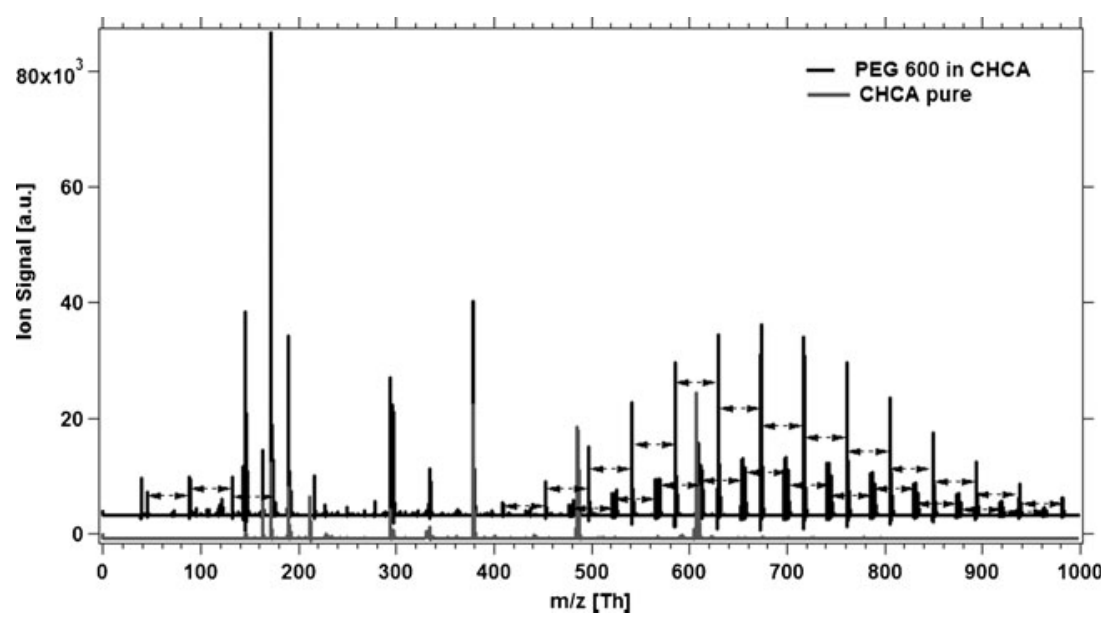

background level and only repeating units in the lower mass range were detectable. As in the case of the plateau, the identification of some of those peaks was easier than during the afterglow due to lower interferences. Also here a dependence on the laser sampling position was observed. Figure 11 shows two different spectra displaying the analyte and the matrix (PEG 600 in CHCA) and the matrix alone (CHCA pure) measured using MALDI in order to distinguish the peaks of the analyte from those of the matrix. It is evident that all the peaks indicated with the arrows (separated by $44 \mathrm{~m} / \mathrm{z}$ ) are from the analyte. Again, the sensitivity was much higher in MALDI.

The major difference in the experimental setup between MALDI and LAGD-TOFMS was the ion generation and extraction. Strategies to improve sensitivity in LAGDTOFMS could therefore include tilting the sample's surface from the horizontal axis connecting the center of the cathode to the sampling orifice. Furthermore, placing the sample directly in front of the sampling orifice with the GD electrodes placed in between could also be an alternative. These geometrical changes of the setup could lead to more efficient ion extraction and therefore contribute to higher sensitivity. Furthermore, an increase in the number of Ar metastable species during the afterglow by increasing the GD current needs to be tested. These changes and further testing of other matrices could make LAGD-TOFMS an interesting technique for the analysis of small organic compounds, complementing the information currently generated by MALDI.

\section{Conclusions}

Ionization of organic analytes was investigated using the different temporal regimes available in a pulsed GD-TOFMS coupled to a laser ablation sampling system. It was found that molecular ions can be preferentially generated in the afterglow compared to the powered phase. The structural information, however, is more easily retrievable in the powered phase due to the harder ionization and increased fragmentation. Fragment identification is also facilitated in the powered regime by the comparatively low signal intensity of the matrix and GD background. Although similar "tunability" is not available in MALDI instruments unless a tandem TOF setup is employed [40], sensitivity achieved in MALDI is significantly higher. However, if an analyte is poorly ionized by a particular matrix in MALDI, LAGD may provide structural and molecular information, which has been demonstrated (e.g., oleic acid).

The measurements performed by LAGD provide some indications that the ionization behavior is less matrixdependent which might be based on the fact that ionization is separated from the vaporization process. However, further studies on the matrix dependence will be required and are currently carried out in our laboratory.

Acknowledgments The authors would like to thank the European Community for financial support through GLADNET, a Marie CurieRTN within the FP 6 , and TOFWERK AG for providing the TOF instrument. The authors also would like to thank Dr. R. Knochenmuss for the discussion of the results, and L. Bertschi (MS service, D$\mathrm{CHAB}$ ) for the MALDI measurements. We also like to thank $H$. Longerich and two anonymous reviewers for critical and constructive comments which helped to improve the manuscript.

\section{Appendix A}

A.1 Chemical structures of the compounds

Here below (Fig. 12) are the chemical structures of the organic molecules used in this study. These structure's formulas were produced using Symyx Draw 4.0.

\section{A.2 MALDI spectrum of CHCA and oleic acid}

A mass spectrum measured with MALDI concerning CHCA alone and mixed with oleic acid is in Fig. 13. 
Fig. 12 Structural formulas of the analytes measured<smiles>N#C/C(=C\c1ccc(O)cc1)C(=O)O</smiles>

CHCA

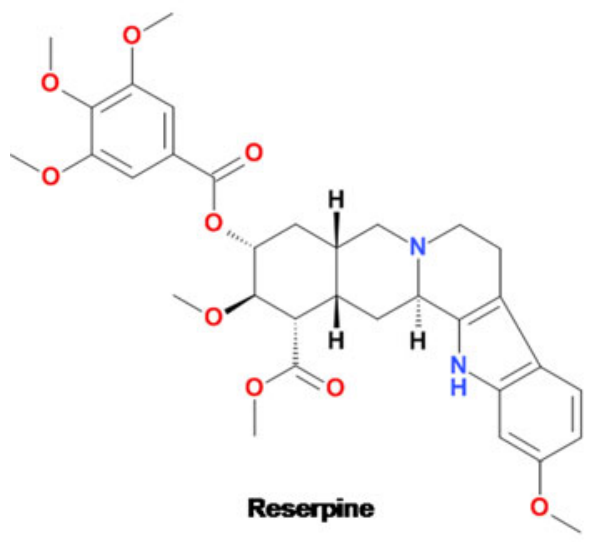<smiles>C[C@@H](CCCC(N)=O)C(=O)N1CCC[C@H]1C(=O)N1CCC[C@H]1C(=O)NCC(=O)N[C@@H](Cc1ccccc1)C(=O)N[C@@H](C[18OH])C(=O)N1CCCC1C(=O)O</smiles>

Bradykinin Fragment 1-7

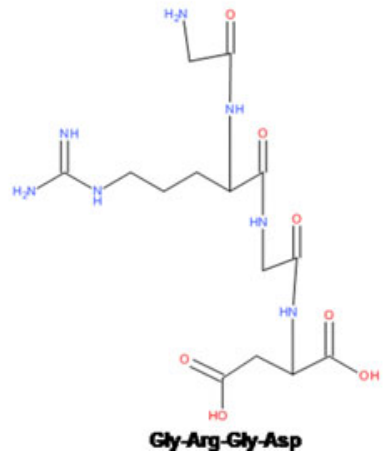

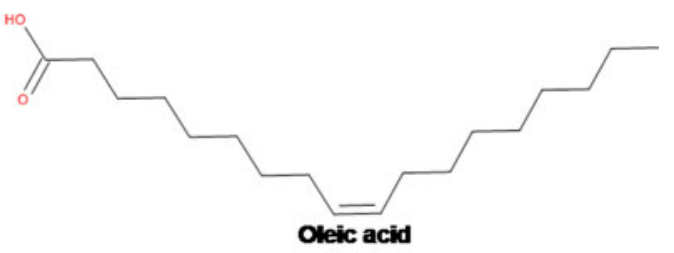

Oleic acid
Ghy-Arg-Ghy-Asp

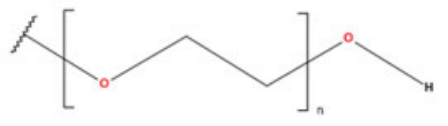

Repeating unit of PEG 600
Fig. 13 Full mass spectra of oleic acid in CHCA (oleic acid and $\mathrm{CHCA}$ ) and of the matrix alone (CHCA pure)

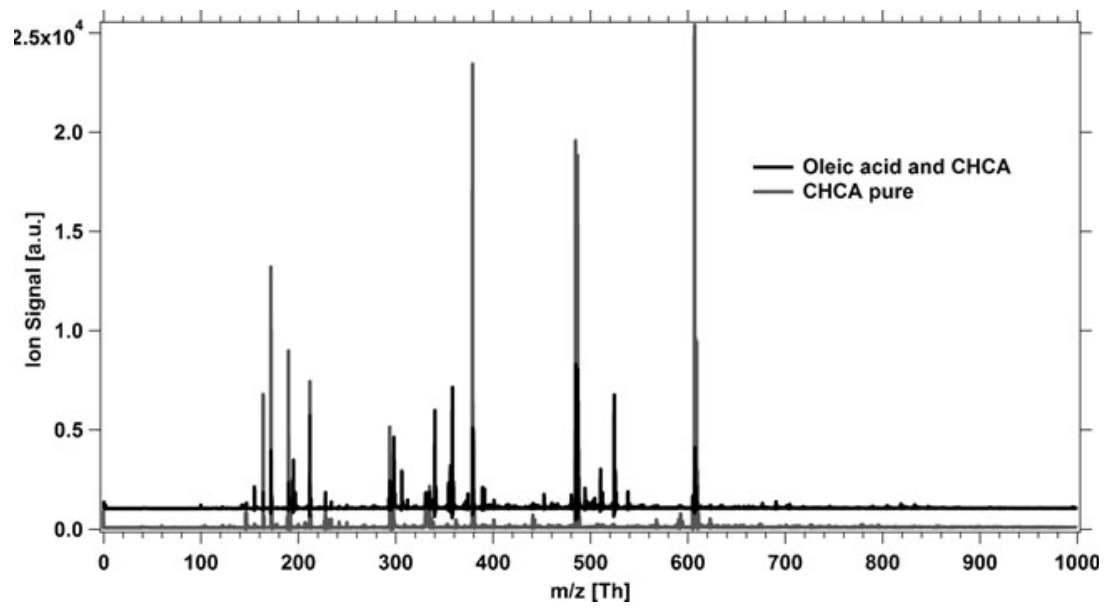




\section{References}

1. Krull IS (1991) Trace metal analysis and speciation. Elsevier, New York

2. Sanz-Medel A (1998) Spectrochim Acta B 53:197-211

3. Ng JC, Johnson D, Imray P, Chiswell B, Moore MR (1998) Analyst 23:929-933

4. Gordon BM, Jones KW (1991) In: Subramanian KS, Iyengar GV, Okamoto K (eds) Biological trace element research, ACS Symposium Serie, Vol. 445, American Chemical Society, Washington, DC

5. Caruso JA, Wuilloud RG, Altamirano JC, Harris WR (2006) J Toxicol Environ Health 9:41-61

6. Waddell R, Lewis C, Hang W, Hassell C, Majidi V (2005) Appl Spectrose 40:33-69

7. Cristoni S, Bernardi LR (2004) Expert Rev Proteomics 1:469-483

8. Siuzdak G (1994) Proc Natl Acad Sci USA 91:11290-11297

9. Ray SJ, Andrade F, Gamez G, McClenathan D, Rogers D, Schilling G, Wetzel W, Hieftje GM (2004) J Chromatogr A 1050:3-34

10. Sola-Vazquez A, Costa-Fernandez JM, Pereiro M, Sanz-Medel A (2011) Analyst 136:246-256

11. Jakubowski N, Dorka R, Steers E, Tempez A (2007) J Anal Atom Spectrom 22:722-735

12. Martin A, Pereiro R, Bordel N, Sanz-Medel A (2007) J Anal Atom Spectrom 22:1179-1183

13. Jackson GP, Lewis CL, Doorn SK, Majidi V, King FL (2001) Spectrochim Acta B 56:2449-2464

14. Yang CL, Ingeneri K, Harrison WW (1999) J Anal Atom Spectrom 14:693-698

15. Pan CK, King FL (1993) J Am Soc Mass Spectrom 4:727-732

16. Majidi V, Moser M, Lewis C, Hang W, King FL (2000) J Anal Atom Spectrom 15:19-25

17. Steiner RE, Lewis CL, Majidi V (1999) J Anal Atom Spectrom 14:1537-1541

18. Lewis CL, Moser M, Dale DE, Hang W, Hassell C, King FL, Majidi V (2003) Anal Chem 75:1983-1996
19. Fliegel D, Fuhrer K, Gonin M, Günther D (2006) Anal Bioanal Chem 386:169-179

20. Tarik M, Günther D (2010) J Anal Atom Spectrom 25:14161423

21. Robertson-Honecker JN, Zhang N, Pavkovichab A, King FL (2008) J Anal Atom Spectrom 23:1508-1517

22. Zhang N, King FL (2009) J Anal Atom Spectrom 24:1489-1497

23. Belkin M, Olson LK, Caruso JA (1997) J Anal Atom Spectrom 12:1255-1261

24. Gibeau TE, Marcus RK (2000) Anal Chem 72:3833-3840

25. Dreisewerd K (2003) Chem. Rev 103:395-425

26. Knochenmuss R (2006) Analyst 131:966-986

27. Tarik M, Lotito G, Whitby JA, Koch J, Fuhrer K, Gonin M, Michler J, Bolli JL, Günther D (2009) Spectrochim Acta B $64: 262-270$

28. Hidaka H, Hanyu N, Sugano M, Kawasaki K, Yamauchi K, Katsuyama T (2007) Ann Clin Lab Sci 37:213-221

29. Kinumi T, Saisu T, Takayama M, Niwa H (2000) J Mass Spectrom $35: 417-422$

30. Shroff R, Svatos A (2009) Rapid Commun Mass Spectrom 23:2380-2382

31. Schaiberger AM, Moss JA (2008) J Am Soc Mass Spectrom 19:614-619

32. Schiller J, Süss R, Fuchs B, Müller M, Petkovic M, Zschörnig O, Waschipky H (2007) Eur Biophys J 36:517-527

33. NIST Chemistry WebBook. Available at http://webbook.nist.gov/ chemistry/ Accessed 19 July 2011

34. Ratliff P, Harrison WW (1995) Appl Spectrosc 49:863-871

35. Ratliff P, Harrison WW (1994) Spectrochim Acta B 49:17471757

36. McCombie G, Knochenmuss R (2004) Anal Chem 76:4990-4997

37. Gusev A, Wilkinson W, Proctor A, Hercules D (1995) Anal Chem 67:1034-1041

38. Vorm O, Roepstorff P, Mann M (1994) Anal Chem 66:3281-3287

39. Cohen S, Chait B (1996) Anal Chem 68:31-37

40. Gogichaeva NV, Williams T, Alterman MA (2007) J Am Soc Mass Spectrom 18:279-284 